\title{
Intelligent security system detects the hidden objects in the smart grid
}

\author{
Ammar Wisam Altaher', Abdullah Hasan Hussein ${ }^{2}$ \\ ${ }^{1}$ Al-Furat Al-Awsat Technical University, Technical Collage of Management, Iraq \\ ${ }^{2}$ Imam Al-kadhum University College, Iraq
}

\begin{abstract}
Article Info
Article history:

Received Jul 1, 2019

Revised Jan 14, 2020

Accepted Feb 8, 2020

\section{Keywords:}

Image analysis

Image fusion

Infrared image

Object detection

ABSTRACT

Monitoring the general public gathered in large numbers is one of the most challenging tasks faced by the law and order enforcement team. There is swiftly demand that has inbuilt sensors that can detect the concealed weapon, from a standoff distance the system can locate the weapon with very high accuracy. Objects that are obscure and invisible from human vision can be seen vividly from enhanced artificial vision systems. Image Fusion is a computer vision technique that fuses images from multiple sensors to give accurate information. Image fusion using visual and infrared images has been employed for a safe, non-invasive standoff threat detection system. The fused imagery is further processed for specific identification of weapons. The unique approach to discover concealed weapons based on DWT in conjunction with Metaheuristic algorithm Harmony Search Algorithm and SVM classification is presented. It firstly uses the traditional discrete wavelets transform along with the hybrid Hotline transform to obtain a fused imagery. Then a heuristic search algorithm is applied to search the best optimal harmony to generate the new principal components of the registered input images which is later classified using the $\mathrm{K}$ means support vector machines to build better classifiers for concealed weapon detection. Experimental results demonstrate the hybrid approach which shows superior performance.
\end{abstract}

Copyright $(2020$ Institute of Advanced Engineering and Science. All rights reserved.

\section{Corresponding Author:}

Ammar Wisam Altaher,

Department of Iinformation Technology,

Technical Collage of Management, Iraq.

Email: dr.ammar@atu.edu.iq

\section{INTRODUCTION}

The recent times have seen a lot of political upheavals and public unrest across the world. Safety and security of public life and public assets such as [1] airport, shopping mall, railway, bus stations have been constantly under threat by self-employed threat organizations for their vested interest. In such situations, the most challenging task of the law and order enforcement would be to perform unrestricted screening and safety surveillance system [2,3] in all public assets with respect to human privacy and with a minimum ratio of the fail cause of related with people life. There is an argent and swiftly demand the need for intelligent surveillance and security systems that have and support inbuilt sensors which any hidden explosive or weapon from a standoff distance. The new intelligent system is a revolution in international security as it can discover future terror threats and it is of paramount importance to the law and order enforcement team who allocate significantly Investment annually to safeguard the innocent lives from new and emerging threats. The burning issue faced by the law and enforcement personnel is identifying the weapons covered with fabrics concealed under human clothing [2]. The detection system can be very necessary to prevent the real threats to the society, the public places can be monitored manually or by a specific gate to detect the dangerous materials. The objective of this work is to design and develop an automated concealed weapon detection system based on image analysis processing and machine-learning principles. The proposed future work supposed to use 
image processing algorithms to combine both of data-set (infrared and visual) images such as image fusion algorithm [3-5]. Which can define it is image processing algorithm can combine images with the same dimensional and create a new image worthy and can use it to detect hidden object after analysis it.

\section{RELATED WORK}

Off late, the security major in the domain of surveillance and detection system has received a maximum boost and reflecting to provide innovative automatic detection to swiftly naturalize dangers proposed by international terrorism. The problem has escalated post the September of 2001 aerial attack on the world trade center. The NIJ lead the work in the right direction and [6] the guidelines issued by national institute justice in 2001 provides a comprehensive summary of image detection and pinpoints multi aspects of the sensor process. Such as illumination, vicinity, transferability, and manageability, due to the fact that several tiny VLC hardware has been supported with Visible light cameras (VLC) employ sensors aspects [7] Furthermore, both lighting sensing in a Camera system and photo-sensitive systems have employed to VLC receivers [8]. Light beams have a major factor to aid any extract data by VLC receivers [9]. While there are several main temperature variations that use for human body temperature, for example, temperature and Infrared (IR) cameras [10] temperature ranges required by different applications and IR cameras are one of the main technology used. But, a critical issue for IR somehow becomes slow in general. Thus, researchers try to have temperature variations to optimal results by tuning a dynamic range of IR sensors [11]. Nowadays, our world facing a high-security conflict-which needs more attention in vital organizations such as airports and military zone area So, starting from developing a full-body camera scanner to open research for a weapon the detection system is still consumption high efforts to mitigate the security issues [12]. One of the feasible solutions that used to detect concealed objectives are Microwave frequencies and issue over microwave frequencies used 2D magnitude images -which reveals full body image and cause privacy concerns and embarrassment [13]. Therefore, many related studies address the privacy issue, for example, a simple scan at distance has proposed for immediately concealed weapons though using ground-breaking technology [14-15]. For semiconductors, the technology world has developed many digital imaging sensors to increase the performance of IR cameras [16]. To provide a uniform image output, modern consumer electronics for capturing image has deployed over electromagnetic spectrum aspects, which used to high substantial internal processing. Sensors highly depend on the light source, and that done through a device have an electrical charge. Digital signal processing techniques used to convert to digital information such as, interpolation algorithms and quantization. The slandered mechanism is to generate an analog image signal and stored in memory [17].

\section{RESEARCH METHOD}

A novel approach proposed is to combine Discrete Wavelet transform with Karhunen Loeve transform also called as Hoteling transform to obtain a fused image from a pair of visual and infra-red images. After the fusion rule, privacy-preserving clustering protocol is to be incorporated along with support vector machines to build better classifiers to detect concealed weapons. The block diagram explains the steps of the algorithm as shown in Figure 1, concealed weapon detection and parameters analysis for both images sensor.

\subsection{Discrete wavelet transform (DWT)}

One of the most powerful mathematical methods to analyze images is a Discrete Wavelet Transform. It decomposes an image and produces a non-redundant output that provides better spatial and spectral lateralization of image transformation, compared with other multi-scale representations such as Gaussian and Laplacian pyramid. DWT decomposes an image into frequency sub-band at a different scale from which it can be reconstructed perfectly using the (IDWT) Inverse Discrete Wavelet Transform. [18] The input image data signal is divided into high and low frequency parts. The low-frequency part contains coarse information of signal whereas the high-frequency part contains information about the edge components. For example, for a given $2 \mathrm{D}$ image an $\mathrm{N}$ level decomposition can be performed resulting in $3 \mathrm{~N}+1$ different frequency bands.At every level of decomposition, we obtain two sets of coefficients, approximation (LL) and detail (HL, LH and HH) coefficients. Firstly, DWT is performed along the vertical direction and then by the horizontal direction. After the first level of decomposition, there are 4 sub-bands: LL1, LH1, HL1, and HH1. For each successive level of decomposition, the LL sub-band or the approximation coefficient of the previous level is used as the input to the next level [19]. The mathematical operation for DWT as follows:

$$
F(\mathrm{c} 1, \mathrm{~d} 1, \mathrm{c} 2, \mathrm{~d} 2) \sum_{m=0}^{M-1} \sum_{n=0}^{N-1}\left(f(m, n) \frac{1}{\sqrt{\mathrm{d} 1 \mathrm{~d} 2}} \varphi\left(\frac{m-\mathrm{c} 1}{d_{1}}, \frac{n-\mathrm{c} 2}{\mathrm{~d} 1}\right)\right)
$$


$f(m, n) \frac{1}{\sqrt{\mathrm{d} 1 \mathrm{~d} 2}}$ It means the wavelet transform coefficient

With respect to $\mathrm{X}$ axes and $\mathrm{Y}$ axes of $\mathrm{c} 1, \mathrm{c} 2, \mathrm{~d} 1, \mathrm{~d} 3$.

To overcome losing the information and dimensionality of images sensors, DWT and components principle analysis demonstrated preservation and protects it from hamper and redundancy problems.

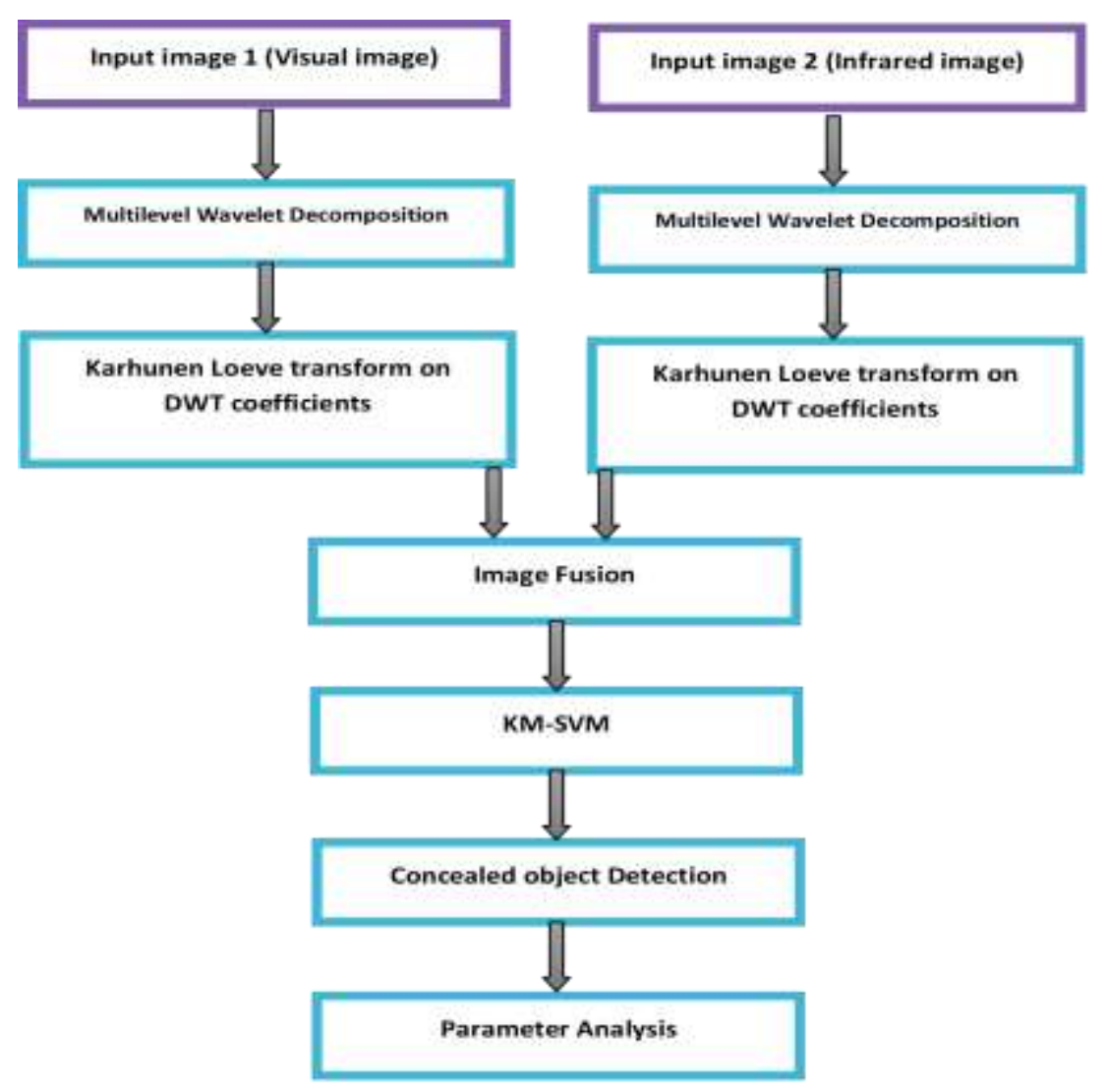

Figure 1. Block diagram of concealed object detection

\subsection{Principal components analysis}

Karl Pearson invented the popular dimensionality reduction algorithm called Principal Component Analysis (PCA) in 1901. This methodology has become more popular to compress an image because of its ability to minimize the input data duplication. This methodology has become more popular to compress an image because of its ability to minimize the input data duplication Additionally, this technology uses a statistical analysis technique in which the supplied data is applied to Eigenspace to maximize the variance and minimize the variables with low Eigenvalues. Hoteling and Karhunen-Loeve transform are alternative names for PCA. PCA is a non-parametric mathematical tool of linear algebra generally applied on large datasets to extract relevant information. It transforms a set of correlated data into an orthogonal set of uncorrelated variables called principal components. It is a lossy transformation and causes performance degradation when PCA is applied to pattern recognition algorithms. In order to represent the original data in a compact fashion, the original ' $\mathrm{M}$ '-valued dataset is reduced to a lower dimension newer revised lower dimension ' $\mathrm{m}$ '-valued data. $90 \%$ of total components are called information content while the rest of the components varies from 2 to $\mathrm{m}$ comprising the reduction variance distribution. PCA finds successful implementation in applications of image recognition and compression such as feature extraction, prediction algorithms, data artifact removal, etc. Since the mathematical basis is linear algebra, many linear models of digital signal processing, information systems, communication systems, and control theory applications can be realized by PCA. The computation of PCA is relatively simple and depends on finding the eigenvectors and eigenvalues. First, the mean of the input data vector is found. Then, the covariance matrix of the vector is calculated and assembled. 
Using the covariance matrix, eigenvalues and eigenvectors are computed forming the principal components in the new data space. The largest eigenvalues represent the majority of the information content. The math formulas of the computations are as follows:

The given dataset $\mathrm{X}$ of dimension $\mathrm{M}$ is transformed to dimension -reduced dataset $\mathrm{Y}$ of dimension $\mathrm{L}$, where $\mathrm{L}<\mathrm{M}, \mathrm{PCA}$ or Hoteling Transform is applied as follows:

$$
\mathrm{Y}=\mathrm{HT}[31]
$$

The experimental mean is shown by

$$
\operatorname{Mean}\left(\overline{X)}=\frac{1}{N} \sum_{n=1}^{N} X_{i}\right.
$$

The formula for covariance is

$$
\operatorname{cov}(X, Y)=\frac{\sum_{n=1}^{N}\left(X_{i-} \bar{X}\right)\left(Y_{i}-\bar{Y}\right)}{N}
$$

To calculate the Eigen values and Eigen vectors:

$$
[A][X]=\lambda X
$$

One of the non-recommended methods is Dimensionality as it effects on the performance of an object-recognition algorithm. PCA and Wavelet are certained to give a better performance than other techniques. Hence, therefore, the unique result shows much better harmony with the algorithm in combination with PCA to displace the redundancy.

\subsection{Image fusion}

Image fusion in the field of computer vision has been explored widely to accommodate varied fusion techniques. Fusion approaches can track targets even under the circumstance of concealed and disguise conditions. To improve combined image works as a strong assessment tool together with IR technology to trace objects. Enhancing the context of the combined image acts as a powerful assessment tool to detect suspicious objects. This makes for a sturdy and reliable system with more complete content that is devoid of any obscurities and uncertainties because all undesirable redundancies and artifacts are successfully repressed and minimized. There are three different methods to use fusion technique depending on the selected level: pixel, viz, decision or features. The main aim is to keep the highest relative information and decrease unwanted data. This can be produced as a fusion-approach with a low pixel result. At the same time, each image has different properties sort out by intensity variation, boundaries, color, and surface smoothness. Image-edge improvement and artificial neural networks are examples of feature-based fusion executions. A technique called Decision-level fusion can combine plenty of algorithms to reach a high-quality as well as a virtual non-artifact fused image. Complicated results deal with different sequential input channels, providing true or false results to move on with the fusion technique [20]. The manipulation of intensity values for each image can be achieved by the spatial-domain method like pixel-minimum fusion, pixel-maximum fusion, filtering, etc. [21].

\subsection{Support vector machine}

Once significant features are extracted from the fused image, a good classification the technique is implemented to identify the suspicious object with in comparison to a reference weapon [22]. This aids the security personnel in identifying and taking appropriate response in case of any threat perceived. The SVM is a pattern recognition algorithm which is regarded as the most powerful tool in the object identification $[23,24]$. The feature vector is classified by using the support vector machine into two classes Weapon detected and No weapon detected.

\subsection{Harmony algorithm working with PCA}

Mathematical harmony algorithm developed for the first time to arrange the musician's work, fix tones of music and find out the best harmony. This algorithm enough works with discrete and continuous value with available three conditions for working. Pitch adjustment, rate [25] equalization and randomization. With memory harmony to pick up old and new harmonies the rate condition of this algorithm fall between 0.7 
and 0.95 with range selection if it is the small rate the selection will be small rate and the storage amount of harmonies will be handoff or all the harmonies will become saved if the large range rate.

\subsection{Normal and hybrid principle components analysis}

Gathering the harmony search and principle components analysis hybrid method, this combination produces best principle components which enhance the image fusion technique. The result computing of HPCA can extraction by DWT of decomposition level 2 .

$$
\operatorname{Mean}\left(\overline{X)}=\frac{1}{N} \sum_{n=1}^{N} X_{i}\right.
$$

Testing mean calculation can be accounting by it

$$
\operatorname{cov}(X, Y)=\frac{\sum_{n=1}^{N}\left(X_{i-} \bar{X}\right)\left(Y_{i}-\bar{Y}\right)}{N}
$$

Testing of covariance mean counting by it

The equation calculate of Eigen and vector machine is

$$
[A][X]=\lambda X
$$

Where $[\mathrm{A}]$ is accounting testing result of wavelet transform coefficient with respect to $\mathrm{X}$ axes and $\mathrm{Y}$ axes of $\mathrm{c} 1, \mathrm{c} 2, \mathrm{~d} 1, \mathrm{~d} 3$.

\section{RESULTS}

The thermal infrared and visual image dataset used in this current paper is about a plain-clothed person concealing a handgun. This is obtained from the repository of Leigh University. To evaluate the novel image fusion technique of KM-SVM hybrid HPCA, a series of simulation experiments were conducted on different image sets. The database was obtained from HTTP: www.ece. Lehigh.edu/SPCRL/IF/cwd/htm Two different approaches were tested. Quality assessment of the image after the fusion algorithm is an important factor to grade the system. The measurement metrics of the image degradation is in comparison with an ideal image or a perfect image. Since image compression techniques such as Discrete Wavelet Transform and Principal Component Analysis transform is applied a considerable dimension reduction and addition redundancy is seen. The amount of degradation is quantified with the following metrics.

HPCA is a method that supplies a powerful improvement of the fusion image for multisensory image data However, the reduction of dimensionality produced a bit of artifact excess HPCA substitute to improve the output results. Through image processing tools classification and identification of the normal and concealed weapon borne person was achieved. The simulation results effectively show the identification of the normal and concealed weapon class, as shown in Table 1 . This method is not computationally heavy so it can rapidly and effectively identify the suspect and alert the law and enforcement personnel to swiftly take action against the person to and ensure the safety and security of the general public. The harmony algorithm consistence with principle components analysis to give the following results as shown in Figure 2-5.

Table 1. The amount degradation of images

\begin{tabular}{clcccccc}
\hline \multirow{2}{*}{$\begin{array}{c}\text { Methodology } \\
\text { adopted }\end{array}$} & $\begin{array}{l}\text { Fused } \\
\text { Images } \\
\text { for CWD }\end{array}$ & $\begin{array}{l}\text { Mean Square } \\
\text { Error }\end{array}$ & $\begin{array}{l}\text { Peak Signal } \\
\text { to Noise } \\
\text { Ratio }\end{array}$ & $\begin{array}{l}\text { Normalized } \\
\text { Absolute } \\
\text { Error }\end{array}$ & $\begin{array}{l}\text { Average } \\
\text { Difference }\end{array}$ & $\begin{array}{l}\text { Cross } \\
\text { correlation }\end{array}$ & $\begin{array}{l}\text { Structural } \\
\text { Content }\end{array}$ \\
\hline HPCA & img1 & 0.0523 & 60.948 & 0.0641 & 0.0641 & 0.9359 & 1.1468 \\
& img2 & 0.0251 & 64.1404 & 0.0382 & 0.0382 & 0.9618 & 1.0823 \\
& img3 & 0.0743 & 59.4199 & 0.0852 & 0.0852 & 0.9148 & 1.1962 \\
& img4 & 0.0504 & 61.11 & 0.0585 & 0.0585 & 0.9415 & 1.1325 \\
& img5 & 0.206 & 54.9925 & 0.2338 & 0.2338 & 0.7662 & 1.6899 \\
PCA & img1 & 0.1788 & 55.6059 & 0.1912 & 0.1912 & 0.8088 & 1.5124 \\
& img2 & 0.1585 & 56.1303 & 0.1709 & 0.1709 & 0.8291 & 1.4136 \\
& img3 & 0.1702 & 55.822 & 0.185 & 0.185 & 0.815 & 1.5157 \\
& img4 & 0.0035 & 72.6888 & 0.0046 & 0.0046 & 0.9954 & 1.0093 \\
& img5 & 0.0406 & 62.047 & 0.0434 & 0.0434 & 0.9566 & 1.0946 \\
\hline
\end{tabular}



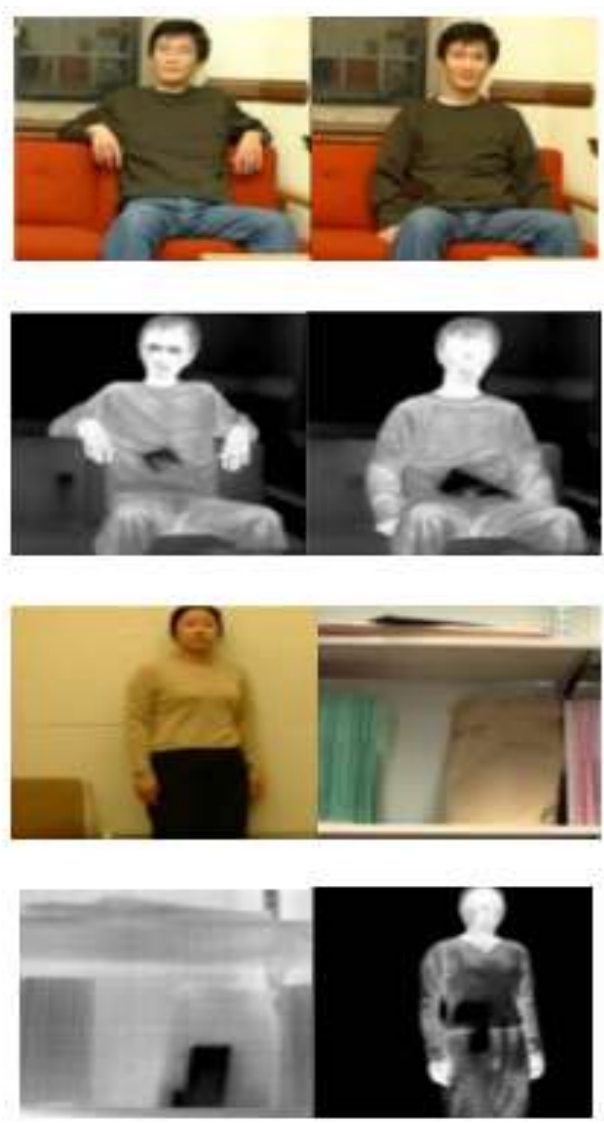

Figure 2. Image dataset using in analysis work

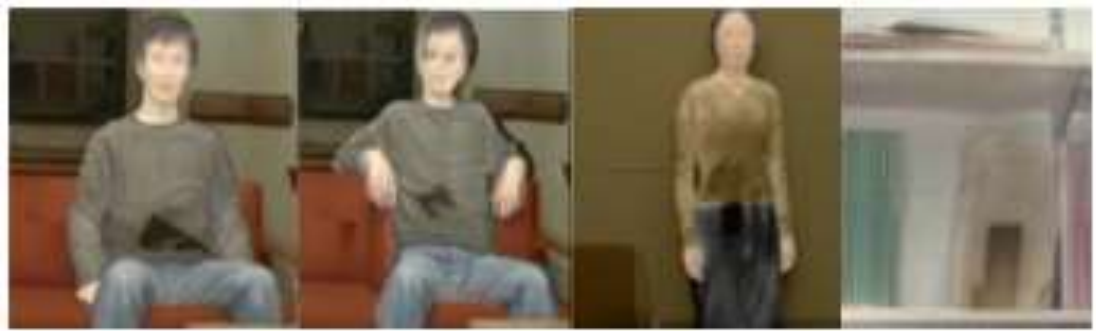

Figure 3. Images fusion dataset of concealed weapon detection

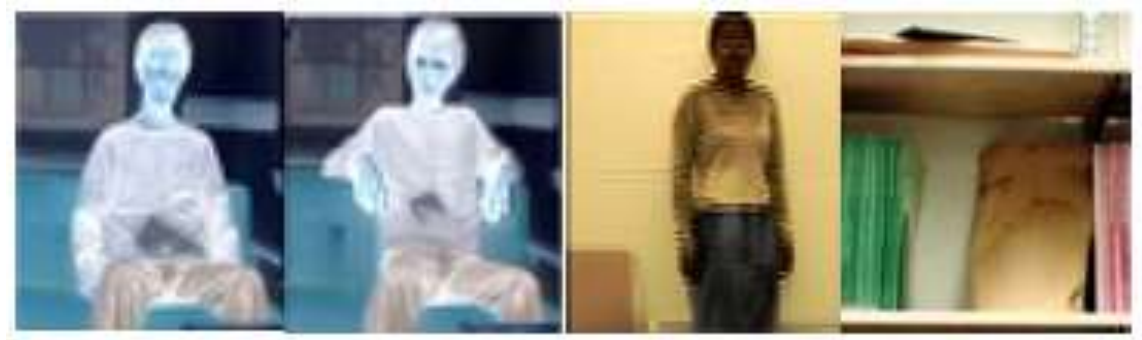

Figure 4. PCA with levels discrete L1, L2, and L3 


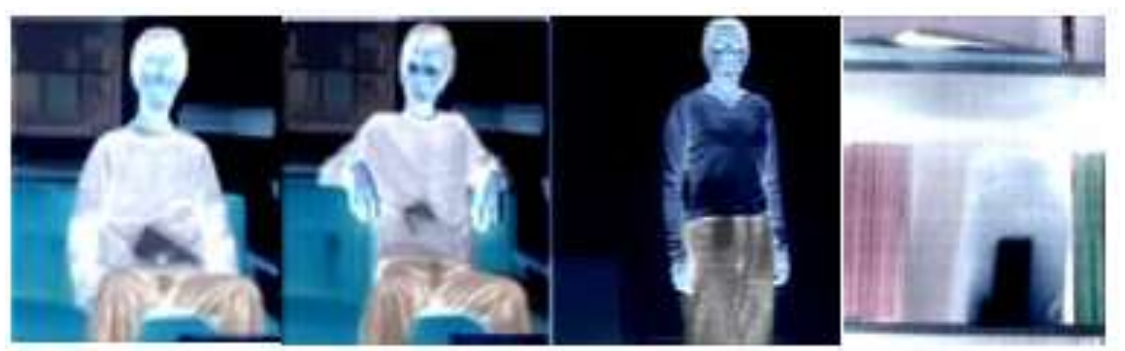

Figure 5. Hybrid component analysis with harmony algorithm

\section{CONCLUSION}

In this current work, a novel method proposed by using SVM, HPCA with harmony algorithm based on analysis of DWT levels decomposing to detect concealed weapon hidden underneath human clothes with respect to privacy from a standoff distance. With computer vision, image fusion techniques support and enhance the images captured by different sensors, the Metaheuristic algorithm using faster and sturdy, the first method of image fusion is carried out with a discrete wavelet transform and PCA combination. The second method of image fusion is carried out with DWT and HPCA combination. The experimental results are computed for the serious of images to provide more strong and active of this algorithm over the DWT and PCA method. The results quantify the claim of the proposed method that the quality of fused output images is enhanced with minimum redundancy.

\section{ACKNOWLEDGEMENTS}

This work was a part of project, to carry out the scientific research activities at the esteemed Al-Furat Al-Awsat Technical University, Najaf, Iraq

\section{REFERENCES}

[1] S. Zhang, C. Wang, S.-C. Chan, X. Wei, and C.-H. Ho, "New object detection, tracking, and recognition approaches for video surveillance over camera network," IEEE Sensors Journal, vol. 15, no. 5, pp. 2679-2691, 2015.

[2] Z. Xue, R. S. Blum, and Y. Li, "Fusion of visual and IR images for concealed weapon detection," pp. 1198-1205.

[3] A. Agurto, et al., "A review of concealed weapon detection and research in perspective," IEEE International Conference on Networking, Sensing and Control, 2007.

[4] M. Kowalski, et al., "Multispectral concealed weapon detection in visible, infrared, and terahertz," Proceedings of SPIE-The International Society for Optical Engineering, 2014.

[5] M. C. Kemp, "Millimetre wave and terahertz technology for detection of concealed threats-a review," 2007 Joint 32nd International Conference on Infrared and Millimeter Waves and the 15th International Conference on Terahertz. Electronics, 2007.

[6] M.-A. Slamani, et al., "Image processing tools for the enhancement of concealed weapon detection," Proceedings 1999 International Conference on Image Processing, pp. 518-522, 1999.

[7] Grobe L, et al, "High-speed visible light communication systems," IEEE Communications Magazine, vol. 51, no. 12, pp. 60-66, 2013.

[8] Tapia G, and Elwany A, "A review on process monitoring and control in metal-based additive manufacturing," Journal of Manufacturing Science and Engineering, vol. 136, no. 6, 2014.

[9] Meola. C, Boccardi. S, and Carlomagno. G. M, "Measurements of very small temperature variations with LWIR QWIP infrared camera," Infrared Physics \& Technology, vol. 72, pp. 195-203, 2015.

[10] Russ. J. C, “The image processing handbook: CRC press," 2016.

[11] Dillon. T. W, and Thomas. D. S, “Airport body scanning: will the American public finally accept?," Journal of Transportation Security, vol. 8, no.1-2, pp. 1-16, 2015.

[12] Sheen. D. M, et al., "Wide-bandwidth, wide-beamwidth, high-resolution, millimeter-wave imaging for concealed weapon detection. SPIE Defense, Security, and Sensing;" International Society for Optics and Photonics,

[13] Brown. K. W, et al., "Infrared concealed object detection enhanced with closed-loop control of illumination by. mmw energy," Google Patents, 2014.

[14] T. Vu, A. Osokin, and I. Laptev. Context-aware CNNs for person head detection. In ICCV, 2015.Ewing. K. J, and Sanghera. J. S., "Extended infrared imaging system," US Patent, 2016.

[15] Ewing. K. J, and Sanghera. J. S., "Extended infrared imaging system," US Patent, 2016.

[16] Bryen. S. D, “Technology Security and National Power,” Winners and Losers: Transaction Publishers, 2015.

[17] Campbell. S. P, et al., "Image sensor alignment in a multi-camera system accelerator architecture," US Patent, 2016. 
[18] A. Cohen, I. Daubechies, and P. Vial, "Wavelets on the interval and fast wavelet transforms," Applied and Computational Harmonic Analysis, vol. 1, no. 1, pp. 54-81, 1993.

[19] U. Qidwai, and C.-H. Chen, "Digital image processing: an algorithmic approach with MATLAB," CRC press, 2009.

[20] C. Pohl and J. L. Van Genderen, "Review article multisensor image fusion in remote sensing: concepts, methods and applications," International Journal of Remote Sensing, vol. 19 no. 5, pp. 823-854, 1998.

[21] G. Piella, "A general framework for multiresolution image fusion: from pixels to regions," Information Fusion, vol. 4, no. 4, pp. 259-280, 2003.

[22] R. Agrawal, and R. Srikant, "Privacy-preserving data mining," pp. 439-450.

[23] H. Duda, and P. Hart, Stork, "Pattern Classification," John Wiley \& Sons, 2001.

[24] K. Julisch, "Clustering intrusion detection alarms to support root cause analysis," ACM Transactions on Information and System Security, vol. 6, no. 4, pp. 443-471, 2003.

[25] X.-S. Yang, "Harmony search as a metaheuristic algorithm," Music-inspired Harmony Search Algorithm, pp. 1-14, 2009.

\section{BIOGRAPHIES OF AUTHORS}

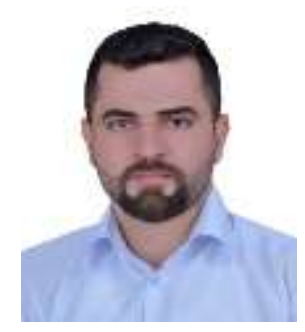

Ammar. Wisam Altaher have expertise in information technology management, $\mathrm{PhD}$ from Kazan federal university /programming technology department / Russia. Master's Degree of Computer Science/Information Technology/Malaysia, Bachelor's Degree of Computer Science/Information Technology/Babylon University, Iraq. Workplace: Al-Furat Al-Awsat Technical University.

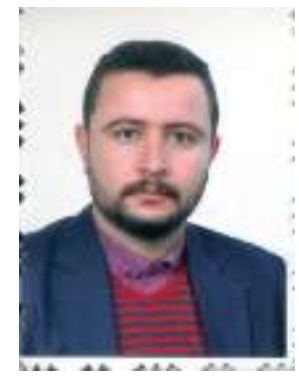

Abdullah Hasan Hussein have expertise in information technology management, $\mathrm{PhD}$ from Kazan federal university /programming technology department / Russia. Master's Degree of Computer Science/Information Technology/Malaysia, Bachelor's Degree of Computer Science/Information Technology/Babylon University, Iraq. Workplace: Imam Al-kadhum University College Najaf Iraq, bdullahhussein87@alkadhum-col.edu 